\title{
Exploratory Analysis of the Effect of Distraction on Driving Behaviour Through a Driving Simulator Experiment
}

\author{
Panagiotis Papantoniou ${ }^{1}$, Constantinos Antoniou ${ }^{2}$, Dimosthenis Pavlou ${ }^{1}$, Eleonora \\ Papadimitriou $^{1}$, George Yannis ${ }^{1}$ and John Golias ${ }^{1}$ \\ ${ }^{1}$ Department of Transportation Planning and Engineering, \\ National Technical University of Athens, Athens, Greece \\ ${ }^{2}$ Laboratory of Transportation Engineering, \\ National Technical University of Athens, Athens, Greece \\ ppapant@central.ntua.gr
}

\begin{abstract}
The objective of this research is the investigation of the effect of different types of distracted driving on driving performance. For this purpose, a driving simulator experiment was carried out at the simulator of the National Technical University of Athens, in which 87 participants were asked to drive under different types of distraction (no distraction, conversation with passenger, mobile phone) and under different traffic conditions (high/low traffic) on urban area. The data collected from the driving simulator experiment include both longitudinal control measures (average speed, headways), lateral control measures (lateral position, standard deviation of lateral position) and the reaction time of the driver at unexpected incidents. In the next step, a descriptive analysis through box plots took place in order to investigate key driving performance parameters. More specifically, participants in the framework of the compensatory behaviour were found to drive at lower speed and with lower speed variability in the distraction trials compared to undistracted driving. Furthermore, regarding the reaction time, results indicate that the distraction mechanism between conversation with the passenger and mobile phone is different and has totally different consequences in the various age groups.
\end{abstract}

Keywords: road safety, driving simulator, urban area, mobile phone

\section{Introduction}

Driver distraction constitutes a basic factor for increased risk for road accidents in Greece and internationally. Although distraction may be considered as a typical part of everyday driving [20], it is reported in the international literature that driver distraction is a contributory factor of road accidents in a proportion ranging from $10-15 \%$ to $30 \%$ [13], whereas driver inattention in general may, together with other factors, affect up to $70 \%$ of road accidents [6]. Within this context, driving simulators have become a widely used tool for examining the impact of driver distraction, with respect to individual driver differences and / or roadway design, as examining distraction causes and impacts in a controlled environment helps provide insights into situations that are difficult to measure in a naturalistic driving environment.

The objective of this research is the investigation of the effect of different types of distracted driving on driving performance. For this purpose, a driving simulator experiment was carried out at the simulator of the National Technical University of Athens, in which 87 participants were asked to drive under different road and traffic conditions (urban/rural area, high/low traffic) and under different types of distraction (no distraction, conversation with passenger, mobile phone). The data collected from the driving simulator experiment include both longitudinal control measures (average 
speed, headways), lateral control measures (lateral position, standard deviation of lateral position) and the reaction time of the driver at unexpected incidents. In the next step, a descriptive analysis through box plots took place in order to investigate these key driving performance parameters.

The paper is structured as follows: In the beginning, a thorough literature review is presented regarding the combined effect of driver distraction (with emphasis on driving simulator experiments examining the effect of mobile phones and conversation with passenger) and other parameters on driving performance. Then, all the methodological steps are presented including the overview of the driving simulator experiment, sample characteristics, driving scenarios, familiarisation and randomisation techniques. Finally, the results are analysed and discussed and some concluding remarks are provided.

\section{Background}

The term distraction has been defined as "a diversion of attention from driving, because the driver is temporarily focusing on an object, person, task or event not related to driving, which reduces the driver's awareness, decision making ability and/or performance, leading to an increased risk of corrective actions, near-crashes, or crashes" [23]. Driver distraction is estimated to be an important cause of vehicle accidents. Although driver distraction can be considered as part of everyday driving, the penetration of various new technologies inside the vehicle, and the expected increase of use of such appliances in the next years, makes the investigation of their influence on the behaviour of drivers and on road safety very essential [18].

Driver distraction factors can be subdivided into those that occur outside the vehicle (external) and those that occur inside the vehicle (in-vehicle). The in-vehicle sources of distraction include the use of mobile phone (either for conversing or for texting), conversation with passengers, smoking, eating or drinking, listening to music and in-vehicle assistance systems (e.g., navigation systems) $[9,15]$ and their effects are largely examined by means of simulator experiments $[8,3]$. For the purpose of this research, an extensive literature review was carried out, presenting driving simulator studies on driver distraction, with emphasis on the effects of mobile phone use and conversation with passengers.

A range of studies have shown that the use of mobile phones has adverse consequences on driver's behaviour and the probability of being involved in an accident. Impairment in situation awareness regarding the surrounding traffic when using hand held cell phones while driving was found by Ma and Kaber [11]. The authors compared the impact of using a hand held cell phone while driving with the use of the adaptive cruise control system and found that the use of cell phone led to a significant reduction in the drivers' situation awareness and a significant increase in the perceived mental workload of the driver.

In a simulator study, Haigney et al., [7] examined the effects on driving performance of engaging in a mobile phone task using hand-held and hands-free mobile phones. Thirty participants completed four simulated drives while completing a grammatical reasoning task designed to simulate a mobile phone conversation. The results revealed that mean speed and the standard deviation of accelerator travel decreased while participants were conversing on the mobile phone. More recent research carried out in a driving simulator by Rakauskas et al., [17] also found that drivers' mean speed decreased and their speed variability increased while carrying out a naturalistic conversation on a mobile phone.

Beede and Kas [2] used a driving simulator to measure the impact on driving of a conversation task on a hands free cell phone and a signal detection task while driving. Driving performance measures in terms of traffic violations, driving maintenance, 
attention lapses and response times were significantly impaired when participants talking on cell phones. Furthermore, conversing on the cell phone and performing the signal detection simultaneously increased the average speed, the number of attention lapses and reduced variability in speed maintenance.

McKnight and McKnight [14] used a video driving sequence that included a total of 45 highway traffic scenes. 150 participants were tested in 5 conditions: Place a cell phone call, engage in a conversation that was either casual or intense, tune a radio or just respond to the traffic scenarios. The authors reported that participants in all conditions failed to respond to traffic events. In particular, the older group of drivers was more vulnerable to multitask demands. The younger group of participants also showed a decrease in their ability to respond to traffic scenarios that was more pronounced in the intense conversation condition.

Using a driving simulator, Strayer et al., [19] found that conversing on a handsfree mobile phone while driving led to an increase in following distance from a lead vehicle and this increase was particularly pronounced under high traffic density conditions.

In Maciej et al., [12] the conversational patterns of 33 drivers and passengers in different in-car settings were compared to a hands-free mobile phone and to a handsfree mobile phone with additional visual information either about the driving situation or the driver. Participants were instructed to have a naturalistic small-talk with a friend and the results of the drivers' speaking behavior showed a reduction of speaking while driving. Moreover, it was shown that, compared to a conversation partner on the mobile phone, a passenger in the car varies his speaking rhythm by speaking more often but shorter.

In the Driving Simulator of the University of Calgary, 40 young drivers encountered motorcycles and pedestrians while making left turns; drivers either drove alone or conversed with an attractive passenger. Measures of looked-but-failed-to-see errors (LBFTS), hazard detection and social factors were analyzed. Higher rates of LBFTS errors and hazard detection occurred while conversing than while driving alone [21].

Charlton [5] compared the driving performance and conversational patterns of drivers speaking with in-car passengers, hands-free cell phones, and remote passengers who could see the driver's current driving situation (via a window into a driving simulator). The results indicated that driving performance suffered during cell phone and remote passenger conversations as compared with in-car passenger conversations and no-conversation controls in terms of their approach speeds, reaction times, and avoidance of road and traffic hazards.

Furthermore, Yannis et al., [22] investigated the effect of different types of conversation on road safety in rural roads. The results suggest that 'simple' and 'complex' conversations are associated with decreased speeds while 'complex' conversations were systematically associated with increased distance from the central axis of the lane, significantly increased reaction times at unexpected incidents and increased accident risk.

\section{Data Collection - Methodology}

Driver distraction research often makes use of driving simulators, as they allow for the examination of a range of driving performance measures in a controlled, relatively realistic and safe driving environment. Driving simulators, however, vary substantially in their characteristics, and this can affect their realism and the validity of the results obtained. Despite these limitations, driving simulators are an increasingly popular tool for measuring and analyzing driver distraction, and numerous studies have been conducted, particularly in the last decade. 


\subsection{Overview of the Experiment}

Within this research, a large driving simulator experiment was including different driving scenarios. The design of the distracted driving scenarios is a central component of the experiment and includes driving in different road and traffic conditions, such as in a rural, urban area with high and low traffic volume. More specifically, this assessment includes an urban driving session with up to six trials and a rural driving session with up to six trials. These trials aim to assess driving performance under typical conditions, with or without external distraction sources. The driving simulator experiment takes place at the Department of Transportation Planning and Engineering of the National Technical University of Athens, where the Foerst Driving Simulator FPF is located. It is a quarter-cab simulator with a motion.

\subsection{Familiarization}

A familiarization session or 'practice drive' is typically the first step of all simulator experiments. The driving simulator provides a "Free Driving" scenario that familiarizes the participants with the demands of an everyday drive. The greater part of the drive is designed in an inter-urban environment, but there is also a short crossing through a small city with traffic lights and junctions. During the familiarization with the simulator, the participant practiced in handling the simulator (starting, gears, wheel handling etc.), keeping the lateral position of the vehicle, keeping stable speed, appropriate for the road environment and braking and immobilization of the vehicle. When all criteria mentioned above were satisfied (there was no exact time restriction), the participant moved on to the next phase of the experiment.

\subsection{Driving at the Simulator}

After the practice drive, each participant drives the two sessions (approximately 20 minutes each). Each session corresponds to a different road environment:

- $\quad$ A rural route that is $2.1 \mathrm{~km}$ long, single carriageway and the lane width is $3 \mathrm{~m}$, with zero gradient and mild horizontal curves.

- An urban route that is $1.7 \mathrm{~km}$ long, at its bigger part dual carriageway, separated by guardrails, and the lane width is $3.5 \mathrm{~m}$. Moreover, narrow sidewalks, commercial uses and parking are available at the roadsides.

Within each road / area type, two traffic scenarios and three distraction conditions are examined in a full factorial within-subject design. The distraction conditions examined concern undistracted driving, driving while conversing with a passenger and driving while conversing on a mobile phone.

The traffic scenarios are:

- $\quad$ QL: Moderate traffic conditions - with ambient vehicles' arrivals drawn from a Gamma distribution with mean $\mathrm{m}=12 \mathrm{sec}$, and variance $\sigma^{2}=6 \mathrm{sec}^{2}$, corresponding to an average traffic volume $\mathrm{Q}=300$ vehicles/hour.

- $\quad$ QH: High traffic conditions - with ambient vehicles' arrivals drawn from a Gamma distribution with mean $\mathrm{m}=6 \mathrm{sec}$, and variance $\sigma^{2}=3 \mathrm{sec}^{2}$, corresponding to an average traffic volume of $\mathrm{Q}=600$ vehicles/hour.

Consequently, in total, each session (urban or rural) includes six trials, i.e., six drives of the simulated route. 


\section{Table 1. Within-subject Design Parameters of the Driving Simulator Experiment}

\begin{tabular}{ccccc}
\hline & \multicolumn{3}{c}{ Road Traffic Conditions } \\
& Urban Area & \multicolumn{2}{c}{ Rural Area } \\
\hline Distraction Sources & $Q_{M}$ & $Q_{H}$ & $Q_{M}$ & $Q_{H}$ \\
\hline No Distraction & $\sqrt{ }$ & $\sqrt{ }$ & $\sqrt{ }$ & $\sqrt{ }$ \\
Cell Phone & $\sqrt{ }$ & $\sqrt{ }$ & $\sqrt{ }$ & $\sqrt{ }$ \\
Conversation & $\sqrt{ }$ & $\sqrt{ }$ & $\sqrt{ }$ & $\sqrt{ }$ \\
With Passenger & & & & \\
\hline
\end{tabular}

\subsection{Incidents}

During each trial of the experiment, two unexpected incidents occur at fixed points along the drive (but not at the exact same point in all trials, in order to minimize learning effects). More specifically, incidents in rural area concern the sudden appearance of an animal (deer or donkey) on the roadway, and incidents in urban areas concern the sudden appearance of an adult pedestrian or of a child chasing a ball on the roadway.

\subsection{Randomization}

The first principle of an experimental design is randomization, which is a random process of assigning treatments to the experimental units. The random process implies that every possible allotment of treatments has the same probability. An experimental unit is the smallest division of the experimental material and a treatment means an experimental condition whose effect is to be measured and compared. The purpose of randomization is to remove bias and other sources of extraneous variation, which are not controllable. Another advantage of randomization (accompanied by replication) is that it forms the basis of any valid statistical test [4]. Hence the treatments must be assigned at random to the experimental units. Randomization is usually done by drawing numbered cards from a well-shuffled pack of cards, or by drawing numbered balls from a well-shaken container or by using tables of random numbers.

\subsection{Sample Characteristics}

The sample of participants is 87 healthy participants aged 18-75 years old. More specifically, 32 young drivers aged 18-34 years old, 33 middle aged drivers aged 3554 years old and 22 older driver aged 55-80 years old consist the sample of the analyses.

\section{Results}

In order to analyse these key measures a descriptive analysis took place through box plots. A box plot (also known as a box-and-whisker chart) is a convenient way to show groups of numerical data, such as minimum and maximum values, upper and lower quartiles, median values, outlying and extreme values.

The spacing between the different parts of the box plot indicates the degree of dispersion (spread) and skewness in the data and identify outliers. More specifically, regarding box plots:

- $\quad$ The line in the middle of the boxes is the median

- $\quad$ The bottom of the box indicates the 25 th percentile. Twenty-five percent of cases have values below the 25th percentile. The top of the box 
represents the 75 th percentile. Twenty-five percent of cases have values above the 75 th percentile. This means that $50 \%$ of the cases lie within the box.

In the present research the following key measures were analysed:

- $\quad$ Average speed - refers to the mean speed of the driver along the route, excluding the small sections in which incidents occurred, and excluding junction areas.

- $\quad$ Standard deviation of average speed - refers to the variability of speed of the driver along the route.

- Reaction time - refers to the time between the first appearance of the event - "obstacle" on the road and the moment the driver starts to brake.

\subsection{Average Speed}

In Figure 1, the average speed of drivers in urban area is presented per distraction factor (no distraction, conversation with passenger, mobile phone use) and per age group (young, middle aged, older). It is observed that young drivers drive in higher speeds regarding middle aged and older drivers, while drivers of all age groups reduce their speed, especially while talking on the mobile phone. Furthermore, while conversing with the passenger, young and middle aged drivers do not change the average speed; however older drivers tend to increase their speed, probably due to a feeling of security that exists due to the passenger.
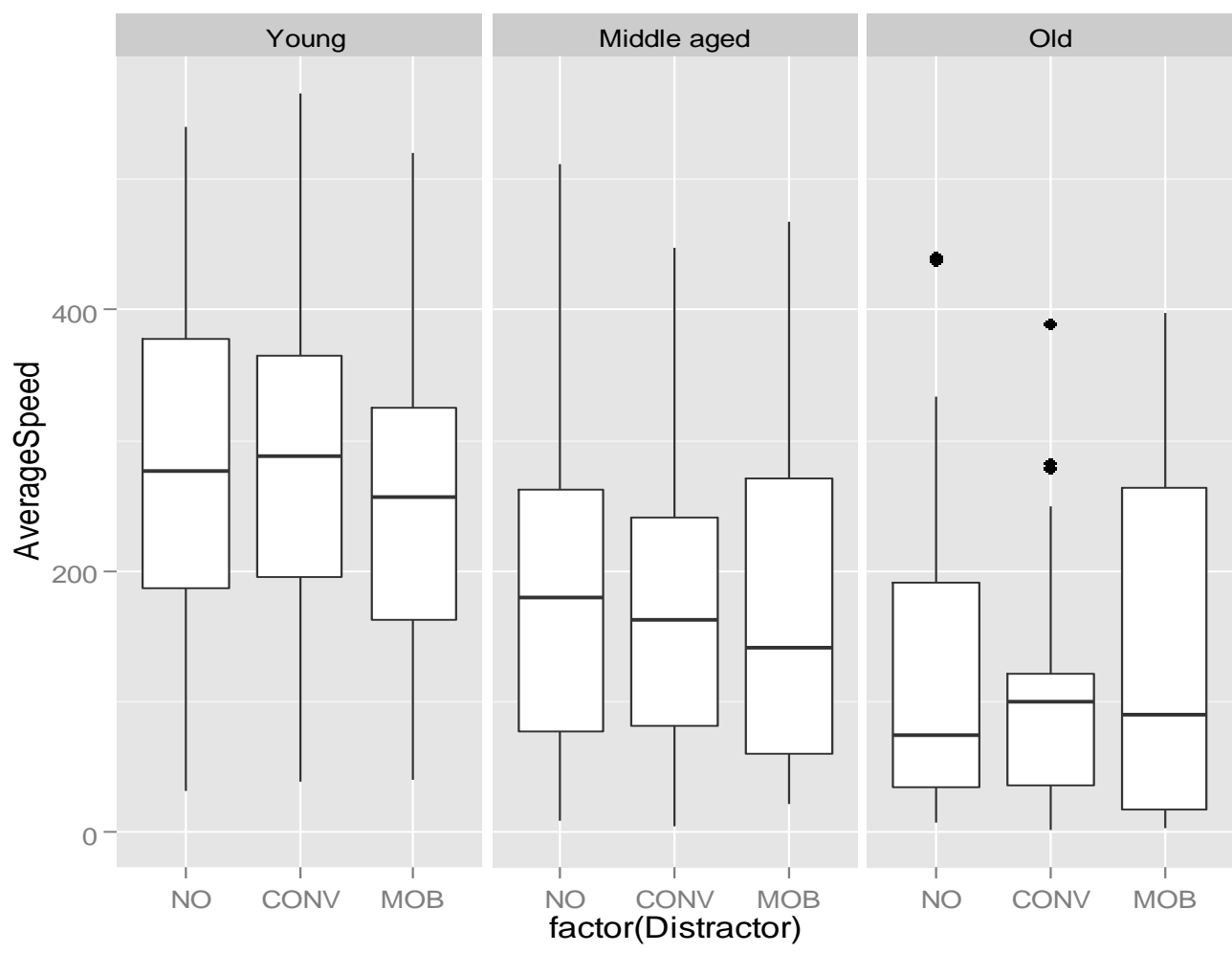

Figure 1. Average Speed per Distraction Factor and Age Group

\subsection{Standard Deviation of Speed}


In Figure 2, the variability of speed (standard deviation of speed) of drivers is presented per distraction factor and per age group and shows that it clearly differs per distraction factor. More specifically, in all age groups drivers have higher speed variability when conversing with the passenger and significant lower speed variability when talking on the mobile phone probably explained by the fact that drivers while talking on the cell phone exhibit what is termed "compensatory behaviour", because the physical presence of a hand-held phone acts as a reminder to the driver of the potential safety threat posed by the use of the phone.

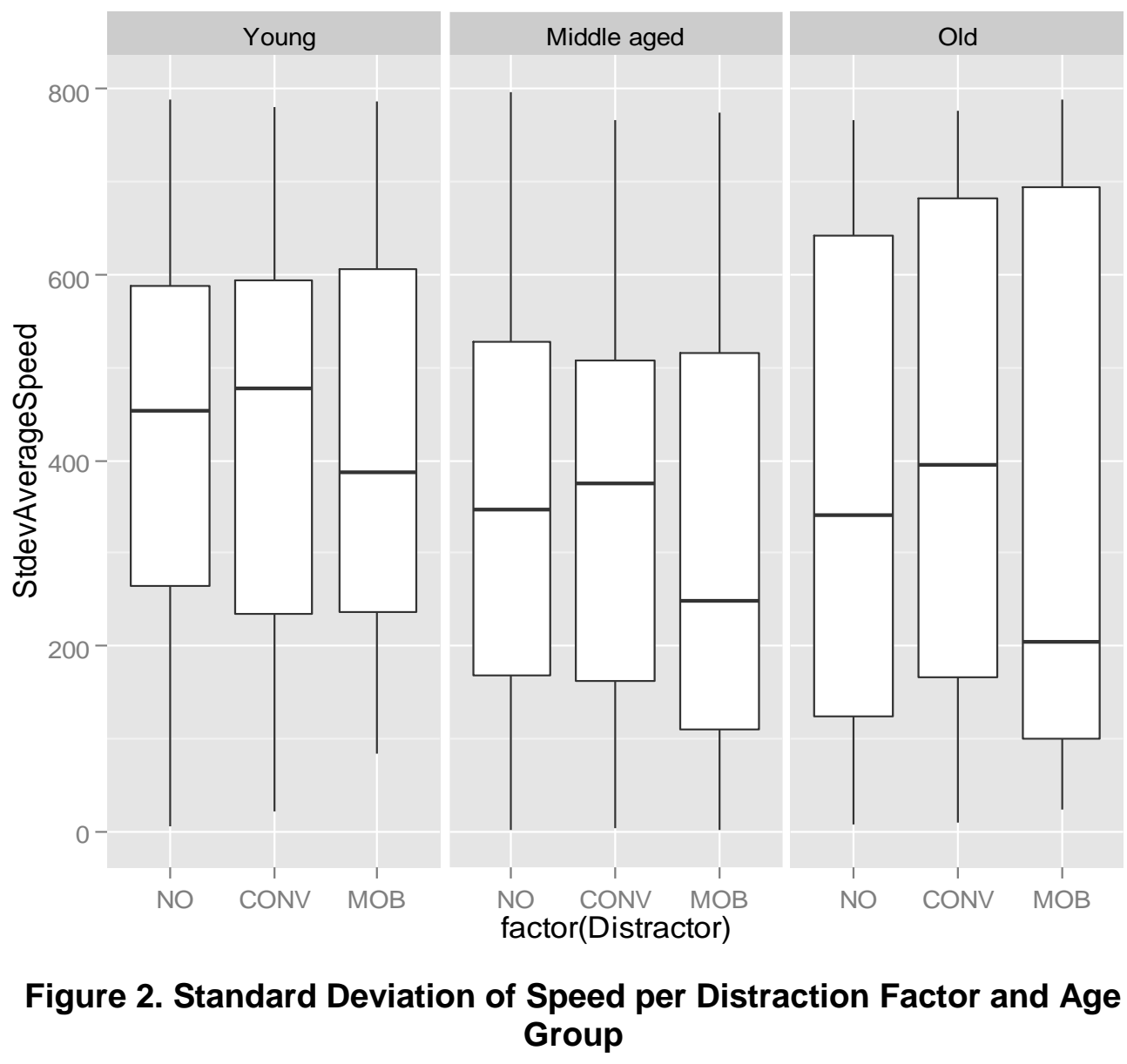

\subsection{Reaction Time}

Figure 3 presents the average reaction time of drivers from two unexpected incidents which occur in each driving trial and concern the sudden appearance of an adult pedestrian or of a child chasing a ball on the roadway. It is clearly observed that, while talking on the mobile phone or conversing with passenger, drivers of all age groups have higher reaction times compared with undistracted driving. Furthermore, it is very interesting that young and middle aged drivers indicate higher reaction times when conversing with the passenger that talking on the mobile phone. On the other hand, older drivers have the worst reaction time when talking on the mobile phone. 


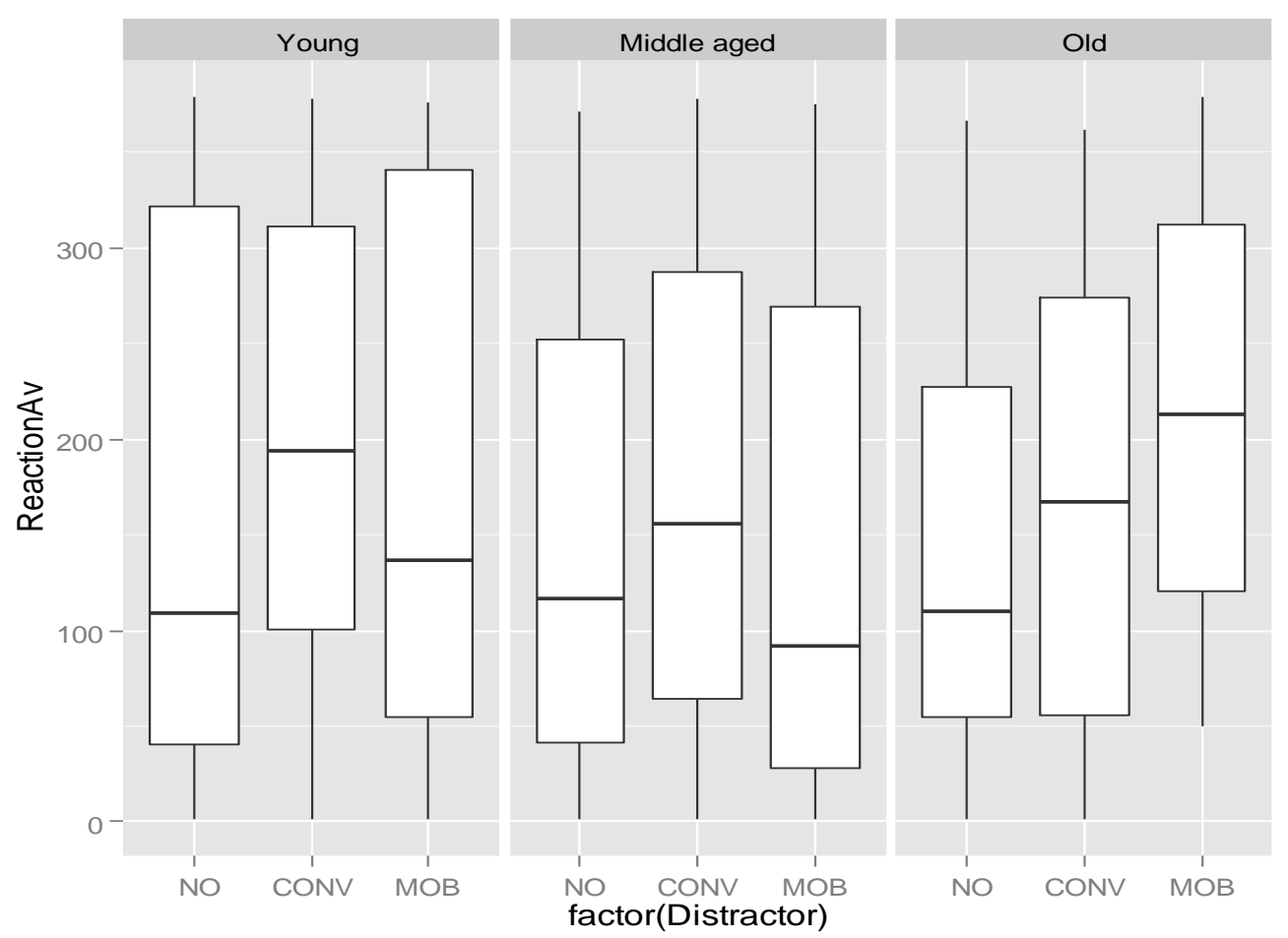

Figure 3. Average Reaction Time per Distraction Factor and Age Group

\section{Discussion}

This paper analysed the driving performance of drivers of different age groups in order to investigate the effect of age and distraction on driving parameters. For this purpose, 87 participants from three different age groups were asked to drive under different types of distraction (no distraction, conversation with passenger, mobile phone use) in urban road environment with low and high traffic volume. In the present research the average speed, the standard deviation of average speed and the reaction time of drivers are analysed and results confirm the initial hypotheses and may reveal differences between driving without any distraction source, conversing with the passenger or talking on the mobile phone for different age groups.

More specifically, regarding average speed results indicate that, as expected, young drivers drive in higher speeds regarding middle aged and older drivers, while drivers of all age groups reduce their speed, especially while talking on the mobile phone. Furthermore, while conversing with the passenger young and middle aged drivers do not change the average speed, however older drivers tend to increase the speed probably due to a feeling of secure that exists due to the passenger.

Furthermore, regarding speed variability, drivers in all age groups have higher speed variability when conversing with the passenger and significant lower speed variability when talking on the mobile phone compared to undistracted driving. This is probably explained by the fact that drivers while talking on the cell phone exhibit again what is termed "compensatory behaviour", because the physical presence of a hand-held phone acts as a reminder to the driver of the potential safety threat posed by the use of the phone.

In this framework, one fundamental question regarding the effect of mobile phone use on driving performance is whether and how drivers self-regulate their driving to compensate for any decrease in attention to the driving task. Compensatory or adaptive behaviour can occur at a number of levels ranging from the strategic (e.g., choosing not to use a mobile phone while driving) to the operational level (e.g., 
reducing speed, reducing speed variability) [16]. At the highest level, drivers can choose to moderate their exposure to risk by choosing not to engage in a potentially distracting task while driving. Research has shown, for example, that older drivers' driving performance is impaired to a greater degree than younger drivers when using a mobile phone and this results in compensatory behaviour at the highest level; many older drivers choose not use a mobile phone while driving $[1,10]$.

On the other hand, the reaction time of the drivers at unexpected incidents exhibited differences between talking on the mobile phone, conversing with the passenger and driving without any distraction. It is clearly observed that, while talking on the mobile phone or conversing with passenger, drivers of all age groups have higher reaction times compared with undistracted driving. Furthermore, it is worth noting that young and middle aged drivers indicate higher reaction times when conversing with the passenger than talking on the mobile phone. This is explained by the fact that, when conversing with the passenger, drivers' glance is out of the road very often while when talking on the mobile phone especially young drivers are familiarized to look at the road continually. On the other hand, older drivers have the worst reaction time when talking on the mobile phone. This is probably explained by the fact that older drivers are not familiarized with the use of mobile phones and cannot operate calls as young or middle aged drivers so mobile phone has a potential negative impact on road safety and may lead to increased accident risk.

It is noted that the above results concern descriptive findings from the first steps of data processing. In the next steps, general linear models should be implemented in order to further investigate the effect of age and distraction on driving parameters. In addition, it would be important to investigate the impact of mobile phone use, not only when the drivers talk on mobile phone using a hand-held device but also when they use a hands-free device, a Bluetooth, or when they type messages.

\section{Acknowledgments}

This paper is based on a research project implemented within the framework of the Operational Program "Education and Lifelong Learning" of the National Strategic Reference Framework (NSRF) - Research Funding Program: THALES, and is cofinanced by the European Union (European Social Fund -ESF) and Greek national funds.

\section{References}

[1] H. Alm and L. Nilsson, "The effects of a mobile telephone task on driver behaviour in a car following situation", Accident, Analysis and Prevention, vol. 27, (1995), pp. 717-715.

[2] K. Beede and S. Kass, "Engrossed in Conversations: The impact on cell phones on simulated driving performance", Accident Analysis and Prevention, vol. 38, (2006), pp. 415.

[3] D. B. Bellinger, B. M. Budde, M. Machida, G. B. Richardson and W. P. Berg, "The effect of cellular telephone conversation and music listening on response time in braking", Transportation Research Part F, vol. 12, no. 6, (2009), pp. 441-451.

[4] L. Boyle, Analytical Tools, In: Fisher, D., Rizzo, M., Caird, J., Lee J., Handbook of Driving Simulation for Engineering, Medicine and Psychology, CRC Press, (2011).

[5] S. Charlton, "Driving while conversing: Cell phones that distract and passengers who react", Accident Analysis and Prevention, vol. 41, (2009), pp. 160-173.

[6] T. A. Dingus, S. G. Klauer, V. L. Neale, A. Petersen, S. E. Lee and J. Sudweeks, "The 100-car naturalistic driving study: Phase II - Results of the 100- car field experiment (report no. DOT HS 810 593)", Washington, DC: National Highway Traffic Safety Administration, (2006).

[7] D. E. Haigney, R. G. Taylor and S. J. Westerman, "Concurrent mobile (cellular) phone use and driving performance: task demand characteristics and compensatory processes", Transportation Research Part F, vol. 3, (2000), pp. 113-121.

[8] T. Horberry, J. Anderson, M. A. Regan, T. J. Triggs and J. Brown, "Driver distraction: The effects of concurrent in-vehicle tasks, road environment complexity and age on driving performance", Accident Analysis and Prevention, vol. 38, (2006), pp. 185-191. 
[9] M. B. Johnson, R. B. Voas, J. H. Lacey, A. S. McKnight and J. E. Lange, "Living dangerously: driver distraction at high speed", Traffic Injury Prevention, vol. 5, no. 1, (2004), pp. 1-7.

[10] D. Lamble, S. Rajalin and H. Summala, "Mobile phone use while driving: public opinions on restrictions", Transportation, vol. 29, (2002), pp. 223-236.

[11] R. Ma and D. Kaber, "Situation awareness and workload in driving while using adaptive cruise control and a cell phone", International Journal of Industrial Ergonomics, vol. 35, (2005), pp. 939-953.

[12] J. Maciej, M. Nitsch and M. Vollrath, "Conversing while driving: The importance of visual information for conversation modulation", Transportation Research Part F, vol. 14, (2011), pp. 512-524.

[13] S. P. McEvoy, M. R. Stevenson and M. Woodward, "The prevalence of, and factors associated with, serious crashes involving a distracting activity", Accident Analysis and Prevention, vol. 39, (2007), pp. 475-482.

[14] A. J. McKnight and A. S. McKnight, "The effect of mobile phone use upon driver attention”, Accident Analysis and Prevention, vol. 25, (1993), pp. 259-265.

[15] D. M. Neyens and L. N. Boyle, "The influence of driver distraction on the severity of injuries sustained by teenage drivers and their passengers", Accident Analysis and Prevention, vol. 40, (2008), pp. 254-259.

[16] L. Poysti, S. Rajalin and H. Summala, "Factors influencing the use of cellular (mobile) phone during driving and hazards while using it", Accident, Analysis and Prevention, vol. 37, (2005), pp. 47-51.

[17] M. E. Rakauskas, L. J. Gugerty and N. J. Ward, "Effects of naturalistic cell phone conversation on driving performance", Journal of Safety Research, vol. 35, (2004), pp. 453-464.

[18] M. L. Regan, J. D. Lee and K. Young, "Driver distraction: theory, effects, and mitigation", CRC Press, (2008).

[19] D. L. Strayer, F. A. Drews and W. A. Johnston, "Cell phone-induced failures of visual attention during simulated driving”, Journal of Experimental Psychology: Applied, vol. 9, no. 1, (2003), pp. 23-32.

[20] J. C. Stutts, D. W. Reinfurt, L. Staplin and E. A. Rodgman, "The role of driver distraction in traffic crashes", Report Prepared for AAA Foundation for Traffic Safety, (2001).

[21] C. White and J. Caird, "The blind date: The effects of change blindness, passenger conversation and gender on looked-but-failed-to-see (LBFTS) errors", Accident Analysis and Prevention, vol. 42, (2010), pp. 1822-1830.

[22] G. Yannis, E. Papadimitriou, X. Karekla and F. Kontodima, "Mobile phone use by young drivers: effects on traffic speed and headways", Transportation Planning and Technology, vol. 4, no. 33, (2010a), pp. 385-394.

[23] K. Young and M. Regan, "Driver distraction: A review of the literature", In: I.J. Faulks, M. Regan, M. Stevenson, J. Brown, A. Porter \& J.D. Irwin (Eds.). Distracted driving. Sydney, NSW: Australasian College of Road Safety, (2007), pp. 379-405.

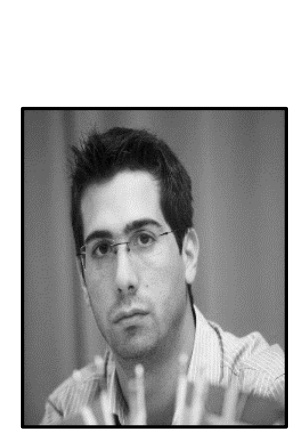

\section{Authors}

Panagiotis Papantoniou, is a Researcher and $\mathrm{PhD}$ Candidate in the field of driver distraction, at the Department of Transportation Planning and Engineering at the School of Civil Engineering of the National Technical University of Athens. In 2008 he graduated from the School of Civil Engineering at the NTUA after having followed the option of Transportation Engineering. He has a Master of Science in the Interdisciplinary - Interdepartmental Program "Water Resources Science \& Technology".

He has participated in 10 research projects, he has published 1 Diploma and 1 master thesis, 29 conference paper, 5 paper in scientific journals, he is a reviewer in a Scientific Journal and in an International Conference and he has attended several scientific conferences.

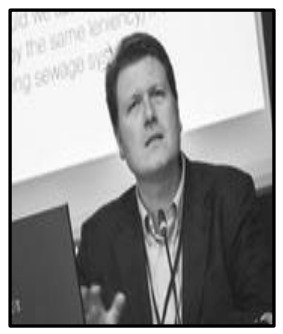

Constantinos Antoniou, is Associate Professor in the National Technical University of Athens (NTUA), Greece. He holds a Diploma in Civil Engineering from NTUA (1995), a MS in Transportation (1997) and a $\mathrm{PhD}$ in Transportation Systems (2004), both from MIT. His research focuses on modelling and simulation of transportation systems, Intelligent Transport 
Systems (ITS), calibration and optimization applications, road safety and sustainable transport systems and in his 17 years of experience he has been involved in a large number of projects, primarily in Europe and the US.

He has authored more than 200 scientific publications, including 65 papers in international, peer-reviewed journals, 125 in international conference proceedings, a book and 15 book chapters. He has held key positions in a number of research projects in Europe, the US and Asia, while he has also participated in a number of consulting projects.

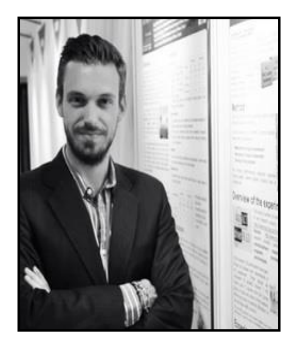

Dimosthenis Pavlou, is a Civil Engineer, PhD Candidate and Researcher at the Department of Transportation Planning and Engineering at the School of Civil Engineering of the National Technical University of Athens.

In 2008, he graduated from the School of Civil Engineering at the NTUA after having followed the option of Structural Engineering. $\mathrm{He}$ has a Master of Science in the Interdisciplinary Interdepartmental Program "Analysis and Design of Earthquake Resistant Structures".

Currently he is a $\mathrm{PhD}$ Candidate and Researcher in the Department of Transportation Planning and Engineering in NTUA in the field of traffic safety. He has participated in 3 research projects, he has published 1 Diploma Thesis and 1 Master Thesis, 17 conference papers, and he has attended several scientific conferences.

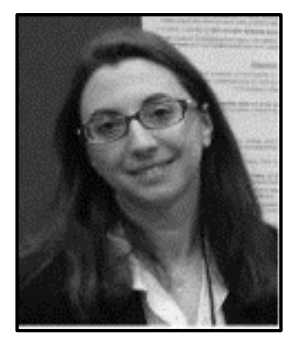

Eleonora Papadimitriou, is Research Associate at the Departmant of Transportation Planning and Engineering of the School of Civil Engineering of the National Technical University of Athens (NTUA). She holds a Civil Engineering Diploma from NTUA (2001), an M.Sc. in Transport from the Ecole National des Ponts et Chaussées (ENPC) in Paris (2003), and a Ph.D. in Transport from NTUA (2010).

She has more than 12 years of experience in several aspects of transportation planning and engineering and her specialisation areas are Road Safety, Traffic Engineering and Transportation Systems.

She has participated in 20 research projects, has more than 95 scientific publications, has participated in more than 35 scientific conferences and seminars, as well as in 5 international scientific committees.

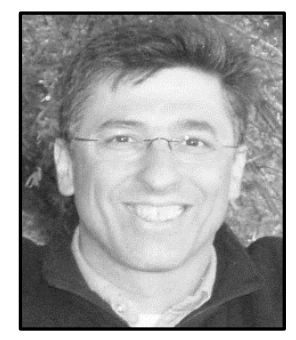

George Yannis, is Professor at the Department of Transportation Planning and Engineering of the School of Civil Engineering of the National Technical University of Athens, teaching Traffic Engineering and Road Safety. He has a Civil Engineering Diploma from NTUA (1987) as well as a DEA 
(1988) and a Doctorat (1993) in Transportation from the Ecole Nationale des Ponts et Chaussées in Paris.

He has a profound and broad understanding of the transportation sector dynamics, through his active involvement for more than 27 years as engineer, academic, advisor and decision maker in all areas of transportation planning and engineering. His specialisation areas are Road Safety, Transportation Planning and Management, Urban Mobility and Intelligent Transportation Systems. He has participated in more than 85 research projects and in 100 projects and studies in Greece and abroad, he has published 320 scientific papers, he has participated in more than 280 scientific conferences and workshops as well as in more than 70 scientific committees in Greece and abroad.

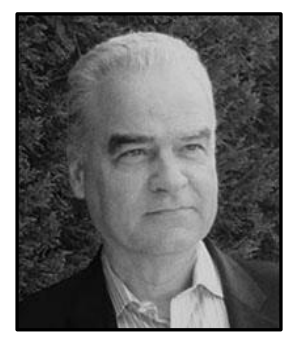

John Golias, is Professor at the Department of Transportation Planning and Engineering and Rector of the National Technical University of Athens. He has a Civil Engineering Diploma from NTUA (1977) as well as a M.Sc. (1979) in Transportation from the Imperial College of Science and Technology, and a Ph.D. (1981) from the University College London. He has been involved in all areas of transportation planning and engineering sector for more than 34 years and his specialisation areas are Traffic Engineering and Road Safety.

He has participated as project coordinator or main researcher in more than 40 research projects in Greece and abroad. He is author of more than 150 scientific papers (65 in peer-review Journals) with more than 200 citations, as well as of 4 books. 